\title{
Coherent Excitation Transfer in a Spin Chain of Three Rydberg Atoms
}

\author{
Daniel Barredo, Henning Labuhn, Sylvain Ravets, Thierry Lahaye, and Antoine Browaeys \\ Laboratoire Charles Fabry, UMR 8501, Institut d'Optique, CNRS, Université Paris Sud 11, \\ 2 avenue Augustin Fresnel, 91127 Palaiseau cedex, France \\ Charles S. Adams \\ Joint Quantum Centre (JQC) Durham-Newcastle, Department of Physics, Durham University, \\ Durham DH1 3LE, United Kingdom
}

(Received 23 September 2014; revised manuscript received 21 November 2014; published 19 March 2015)

\begin{abstract}
We study coherent excitation hopping in a spin chain realized using highly excited individually addressable Rydberg atoms. The dynamics are fully described in terms of an $X Y$ spin Hamiltonian with a long range resonant dipole-dipole coupling that scales as the inverse third power of the lattice spacing, $C_{3} / R^{3}$. The experimental data demonstrate the importance of next neighbor interactions which are manifest as revivals in the excitation dynamics. The results suggest that arrays of Rydberg atoms are ideally suited to large scale, high-fidelity quantum simulation of spin dynamics.
\end{abstract}

DOI: 10.1103/PhysRevLett.114.113002

PACS numbers: $32.80 . \mathrm{Ee}, 03.67 . \mathrm{Bg}, 05.50 .+\mathrm{q}$

Spin Hamiltonians, introduced in the early days of quantum mechanics to explain ferromagnetism, are widely used to study quantum magnetism [1]. Assemblies of interacting, localized spins are a paradigm of quantum many-body systems, where the interplay between interactions and geometry-induced frustration creates a wealth of intriguing quantum phases. Many other phenomena, such as coherent energy transfer, photochemistry, or photosynthesis [2], can also be described using spin Hamiltonians. However, despite this fundamental significance, exact analytical solutions are known only for the simplest cases, and numerical simulations of strongly correlated spin systems are notoriously difficult.

For those reasons, quantum simulation of spin Hamiltonians by controllable systems raises great interest. Recently, various approaches were followed to simulate spin systems using tools of atomic physics [3], such as cold atoms [4-6] or polar molecules [7] in optical lattices, interacting via weak exchange or dipole-dipole interactions, or trapped ions with engineered effective interactions [8-10]. As compared to their condensed-matter counterparts, the spin couplings can be long range, which gives rise to new properties [11-14].

Rydberg atoms are a promising alternative platform for quantum simulation $[15,16]$. In particular, they allow implementing various spin-1/2 Hamiltonians on twodimensional lattices with strong couplings, in the $\mathrm{MHz}$ range $[17,18]$. Rydberg systems interacting through van der Waals interactions can be described by Ising-type

Published by the American Physical Society under the terms of the Creative Commons Attribution 3.0 License. Further distribution of this work must maintain attribution to the author(s) and the published article's title, journal citation, and DOI.
Hamiltonians $H=\sum_{i j} V_{i j} \sigma_{i}^{z} \sigma_{j}^{z}$ where $\sigma^{z}$ is the $z$-Pauli matrix acting in the (pseudo-) spin Hilbert space, and $V_{i j} \sim\left|\boldsymbol{r}_{i}-\boldsymbol{r}_{j}\right|^{-6}$, where $\boldsymbol{r}_{i}$ denotes the position of atom $i$ [17-21]. On the other hand, spin-exchange, or $X Y$, spin Hamiltonians of the form $H=\sum_{i j} V_{i j}\left(\sigma_{i}^{+} \sigma_{j}^{-}+\sigma_{i}^{-} \sigma_{j}^{+}\right)$, where $\sigma^{ \pm}=\sigma^{x} \pm i \sigma^{y}$ are spin-flip operators and $V_{i j} \sim\left|\boldsymbol{r}_{i}-\boldsymbol{r}_{j}\right|^{-3}$, can be realized by using two different Rydberg states, interacting directly via the resonant dipoledipole interaction. However, in this case, only incoherent transfer of excitations has been observed so far, due to the random atomic positions in the ensembles used in experiments [22-27].

In this Letter, we study the coherent dynamics of a spin excitation in a chain of three Rydberg atoms. The dipole-dipole interaction between atoms is given by the $X Y$ Hamiltonian [28]

$$
H=\frac{1}{2} \sum_{i \neq j} \frac{C_{3}}{R_{i j}^{3}}\left(\sigma_{i}^{+} \sigma_{j}^{-}+\sigma_{i}^{-} \sigma_{j}^{+}\right)
$$

where $R_{i j}=\left|\boldsymbol{r}_{i}-\boldsymbol{r}_{j}\right|$ is the distance between atoms $i$ and $j$. We calibrate the spin-spin coupling between two Rydberg atoms by investigating the temporal evolution of two Rydberg atoms prepared in the state $|\uparrow \downarrow\rangle$, as a function of distance $R$ between the atoms, up to $R \simeq 50 \mu \mathrm{m}$. We then use three Rydberg atoms prepared in $|\uparrow \downarrow \downarrow\rangle$ and study the propagation of the excitation through this minimalistic spin chain, observing the effect of long-range hopping of the excitation. The agreement between experimental data and the $X Y$ model without adjustable parameters validates our setup as a future quantum simulator for systems of many spins in arbitrary two-dimensional arrays.

The experimental setup, shown in Fig. 1(a), is detailed in Ref. [30]. Briefly, we focus a red-detuned trapping beam 


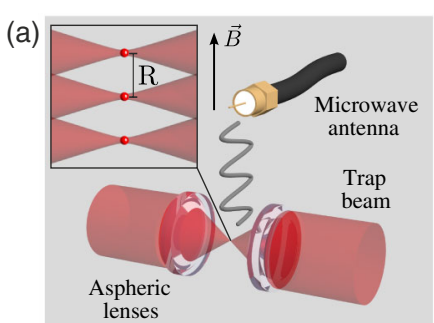

(c)
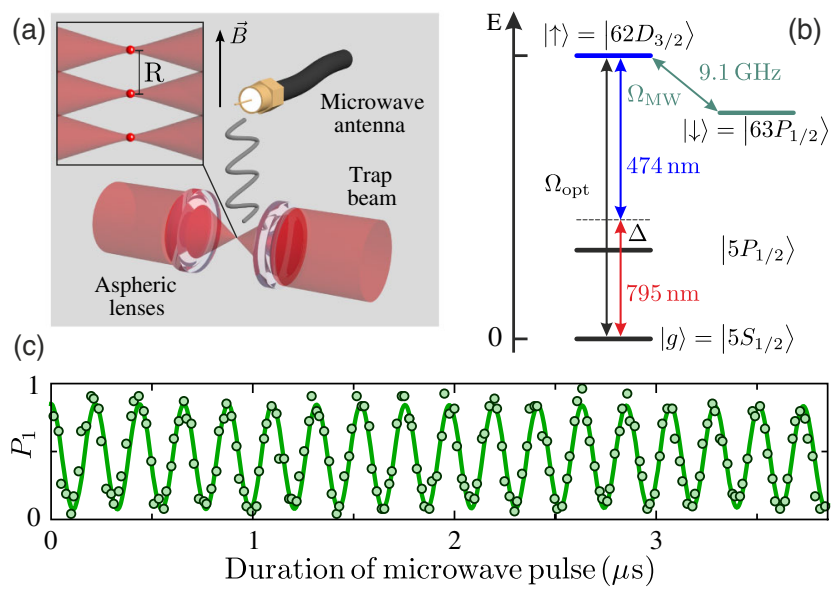

FIG. 1 (color online). (a) Individual ${ }^{87} \mathrm{Rb}$ atoms in microtraps aligned along the quantization axis, defined by a $B=6 \mathrm{G}$ magnetic field. (b) Excitation lasers couple the ground state $|g\rangle=\left|5 S_{1 / 2}, F=2, m_{F}=2\right\rangle$ and the Rydberg state $|\uparrow\rangle=\mid 62 D_{3 / 2}$, $\left.m_{J}=3 / 2\right\rangle$ with an effective Rabi frequency $\Omega_{\text {opt }}$. Microwaves couple $|\uparrow\rangle$ to $|\downarrow\rangle=\left|63 P_{1 / 2}, m_{J}=1 / 2\right\rangle$, with Rabi frequency $\Omega_{\mathrm{MW}}$. (c) Microwave-driven Rabi oscillation of a single atom between $|\uparrow\rangle$ and $|\downarrow\rangle$, yielding $\Omega_{\mathrm{MW}}=2 \pi \times 4.6 \mathrm{MHz}$.

with an aspheric lens into a magneto-optical trap of ${ }^{87} \mathrm{Rb}$, to a waist $\simeq 1 \mu \mathrm{m}$. Multiple traps at arbitrary distances are created by imprinting an appropriate phase on the trapping beam with a spatial light modulator [31]. Because of fast light-assisted collisions in the small trapping volume, at most one atom is present in each trap. The temperature of the trapped atoms is approximately $50 \mu \mathrm{K}$. A 6 G magnetic field defines the quantization axis [32].

We encode the two spin states in the Rydberg states $|\uparrow\rangle=\left|62 D_{3 / 2}, m_{j}=3 / 2\right\rangle$ and $|\downarrow\rangle=\left|63 P_{1 / 2}, m_{j}=1 / 2\right\rangle$ [see Fig. 1(b)]. We trigger an experiment when an atom is detected in each trap. To prepare the atoms in a desired spin state, we first optically pump them in $|g\rangle=\mid 5 S_{1 / 2}$, $\left.F=2, m_{F}=2\right\rangle$. We then switch off the traps to avoid inhomogeneous light shifts, and excite the atoms to $|\uparrow\rangle=\left|62 D_{3 / 2}, m_{J}=3 / 2\right\rangle$ via a two-photon transition (wavelengths 795 and $474 \mathrm{~nm}$, with polarizations $\pi$ and $\sigma^{+}$, respectively), detuned from the intermediate state $\left|5 P_{1 / 2}, F=2, m_{F}=2\right\rangle$ by $\Delta \simeq 2 \pi \times 740 \mathrm{MHz}$. From the $|\uparrow\rangle$ state the atom can be transferred to $|\downarrow\rangle=$ $\left|63 P_{1 / 2}, m_{j}=1 / 2\right\rangle$ using resonant microwaves at $\simeq 9.131 \mathrm{GHz}$, emitted by an antenna outside the vacuum chamber.

To read out the state of an atom at the end of a sequence, we switch on the excitation lasers, coupling only $|\uparrow\rangle$ back to the ground state. We then turn on the dipole traps to recapture ground-state atoms, while atoms in Rydberg states remain untrapped, and detect atoms in $|g\rangle$ by fluorescence. Therefore if we detect an atom in its trap at the end of a sequence, we assume it was in $|\uparrow\rangle$, while a loss corresponds to the $|\downarrow\rangle$ state. We reconstruct all the $2^{N}$ probabilities $P_{i_{1} \ldots i_{k} \ldots i_{N}}$ of having $i_{k}$ atom in trap $k$, with $i_{k}=0$ or 1 , for our $N$-trap system (with $N=1,2$, or 3 ) by repeating the experiment typically 100 times. For instance for $N=3, P_{100}$ is the probability to recapture an atom in trap 1, while recapturing none in traps 2 and 3 . The statistical error on the determination of the probabilities is below 5\%. Figure 1(c) illustrates the coherent spin manipulation for a single atom, by showing Rabi oscillations between $|\uparrow\rangle$ and $|\downarrow\rangle$ : the probability $P_{1}$ to recapture the atom oscillates with a frequency $\Omega_{\mathrm{MW}} \simeq 2 \pi \times 4.6 \mathrm{MHz}$. In $4 \mu \mathrm{s}$, we induce more than 35 spin flips without observing noticeable damping.

We first use two atoms, aligned along the quantization axis, to directly measure the coupling between two spins as a function of their distance. The sequence is shown in Fig. 2(a). We illuminate atom 1 with an addressing beam [33] which induces a $20 \mathrm{MHz}$ light shift, making it off resonant to the global Rydberg excitation. Atom 2 is excited to $|\uparrow\rangle$, and then transferred to $|\downarrow\rangle$ using microwaves. Subsequently, atom 1 is optically excited to the $|\uparrow\rangle$ state with the addressing beam switched off (atom 2 in $|\downarrow\rangle$ is not affected by the Rydberg excitation pulse). We let the system evolve for an adjustable time $\tau$ and read out the

(a)

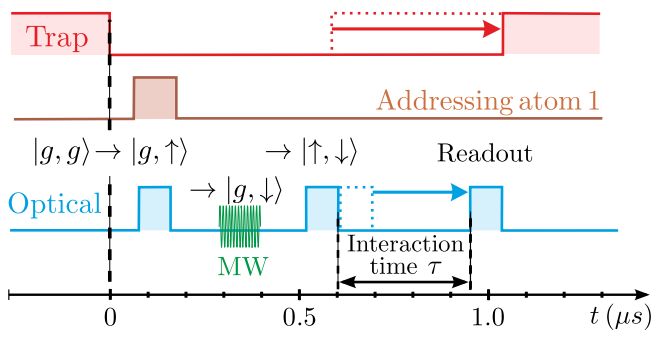

(b)
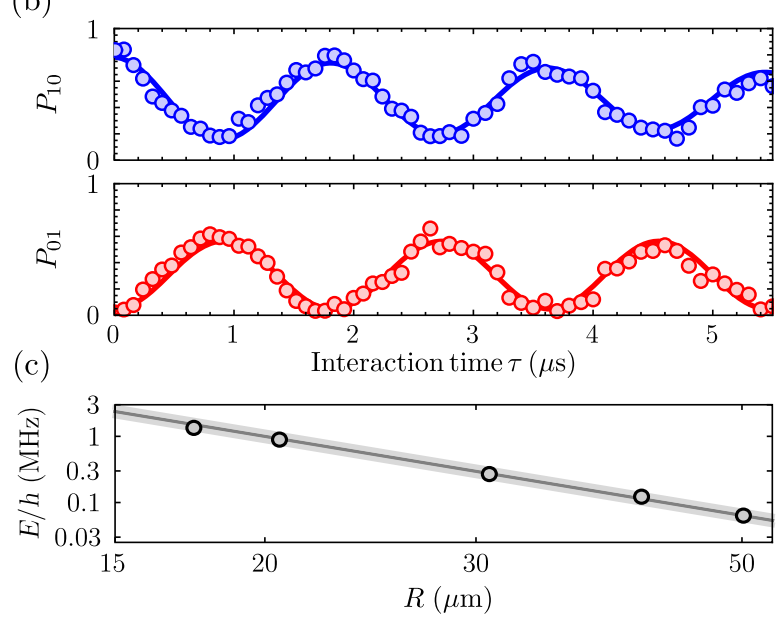

FIG. 2 (color online). (a) Sequence to observe spin exchange between two atoms. (b) Excitation hopping between states $|\uparrow \downarrow\rangle$ (blue disks) and $|\downarrow \uparrow\rangle$ (red disks) of two atoms separated by $R=30 \mu \mathrm{m}$. Solid lines are sinusoidal fits, with frequency $2 E / h$. (c) Interaction energy $E$ (circles) versus $R$. Error bars are smaller than the symbols size. The line shows the theoretical prediction $C_{3} / R^{3}$ with $C_{3}^{\text {th }}=7965 \mathrm{MHz} \mu \mathrm{m}^{3}$. The shaded area corresponds to our systematic $5 \%$ uncertainty in the calibration of $R$. 
final state by deexciting $|\uparrow\rangle$ back to $|g\rangle$. In the absence of experimental imperfections (see [28], Sec. S.3), $P_{10}$ (respectively, $\left.P_{01}\right)$ would give the population of $|\uparrow \downarrow\rangle$ (respectively, $|\downarrow \uparrow\rangle)$ ).

The evolution of $P_{10}(\tau)$ and $P_{01}(\tau)$ for two atoms prepared in $|\uparrow \downarrow\rangle$ separated by $30 \mu \mathrm{m}$ is shown in Fig. 2(b). The spin excitation oscillates back and forth between the two atoms, with a frequency $2 E / h \approx$ $0.52 \mathrm{MHz}$. The finite contrast is essentially due to spontaneous emission via the intermediate $\left|5 P_{1 / 2}\right\rangle$ state during preparation and readout, which limits the oscillation amplitude to about $60 \%$, and, to a lesser extent, to the onset of dipolar interactions during the second excitation pulse [28]. We then repeat the same experiment for several values of the distance $R$ between the atoms, and observe spin-exchange oscillations for distances as large as $50 \mu \mathrm{m}$. Figure 2(c) shows the measured interaction energies as a function of $R$, together with the expected $C_{3} / R^{3}$ behavior (solid line) for the theoretical value $C_{3}^{\text {th }}=7965 \mathrm{MHz} \mu \mathrm{m}^{3}$ of the $C_{3}$ coefficient, calculated from the dipole matrix elements $\left\langle\uparrow\left|\hat{d}_{ \pm 1}\right| \downarrow\right\rangle[30,34]$. A power-law fit to the data (not shown) gives an exponent $-2.93 \pm 0.20$. Fixing the exponent to -3 gives $C_{3}^{\exp }=7950 \pm 130 \mathrm{MHz} \mu \mathrm{m}^{3}$. The agreement between data and theory is excellent.

We now extend the system to a three-spin chain, with a distance $R=20 \mu \mathrm{m}$ between the atoms. The sequence is similar to that in Fig. 2(a) for two atoms, except that we now use microwave transfer for atoms 2 and 3 to prepare $|g \downarrow \downarrow\rangle$. Here, the van der Waals interaction between the two atoms in $|\uparrow\rangle$ is only $\sim 10 \mathrm{kHz}$ for $R=20 \mu \mathrm{m}$, and thus no blockade effect arises during excitation. We then excite atom 1 to prepare $|\uparrow \downarrow \downarrow\rangle$.
We first analyze theoretically the evolution of the system. Assuming that the initial state is $|\psi(0)\rangle=$ $|\uparrow \downarrow \downarrow\rangle$, the dynamics induced by the $X Y$ Hamiltonian (1), which conserves the total magnetization $\sum_{i} \sigma_{i}^{z}$, occurs within the subspace spanned by $\{|\uparrow \downarrow \downarrow\rangle,|\downarrow \uparrow \downarrow\rangle,|\downarrow \downarrow \uparrow\rangle\}$. Figures 3(a) and 3(b) show the calculated dynamics of the spin excitation, which moves back and forth between the extreme sites. Figure 3(a) corresponds to the case where only nearest-neighbor interactions are retained in (1). Periodic, fully contrasted oscillations at a frequency $\sqrt{2} C_{3} / R^{3}$ are expected for the population of the extreme sites, while the population of $|\downarrow \uparrow \downarrow\rangle$ oscillates twice as fast between 0 and 1/2. In contrast, in Fig. 3(b), the full Hamiltonian (1) is simulated, including the interaction between extreme sites. One observes a clear signature of this long-range coupling, as the dynamics now becomes aperiodic for the populations of $|\uparrow \downarrow \downarrow\rangle$ and $|\downarrow \downarrow \uparrow\rangle$. The interplay of the couplings $C_{3} / R^{3}$ and $C_{3} /\left(8 R^{3}\right)$ between nearest- and next-nearest neighbors makes the eigenvalues of (1) incommensurate. The back-and-forth exchange of excitation is thus modulated by a slowly varying envelope due to the beating of these frequencies.

Figure 3(c) shows the experimental results for $P_{100}, P_{010}$, and $P_{001}$ (symbols). We observe qualitative agreement with Fig. 3(b), in particular the "collapse and revival" in the dynamics showing the effects of the long-range coupling. However, one notices differences with the ideal case: (i) the preparation is imperfect, as one starts with a significant population in $|\downarrow \uparrow \downarrow\rangle$, (ii) this, together with imperfect readout [28], reduces the overall amplitude of the oscillations, and (iii) the oscillations show some damping, which becomes significant for $\tau \geq 4 \mu \mathrm{s}$.
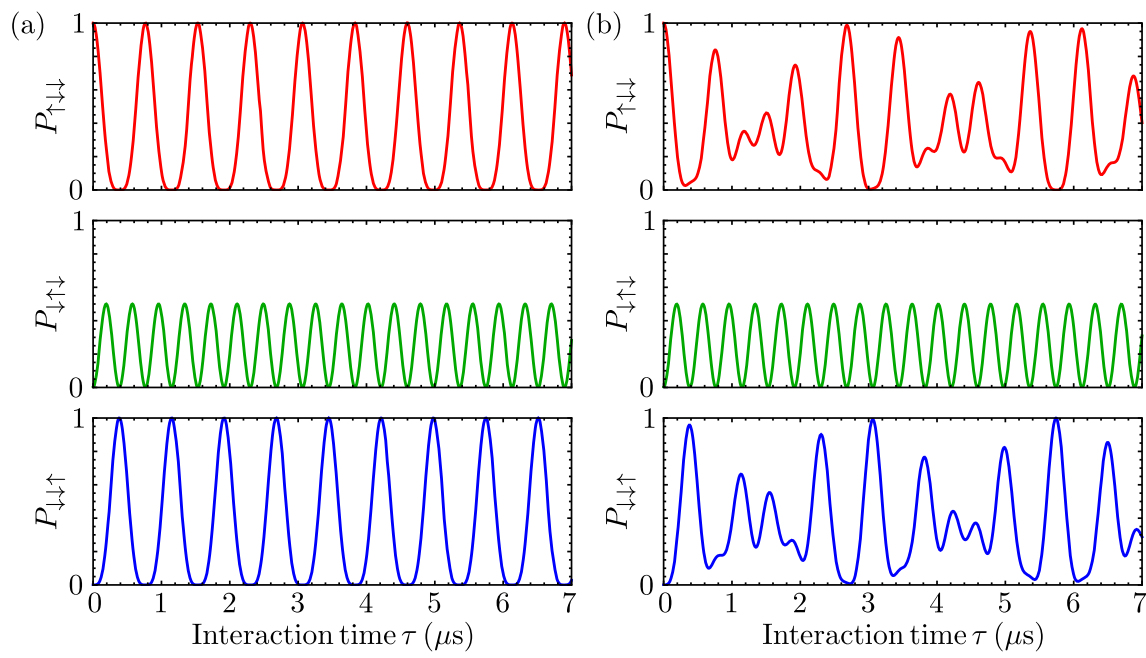
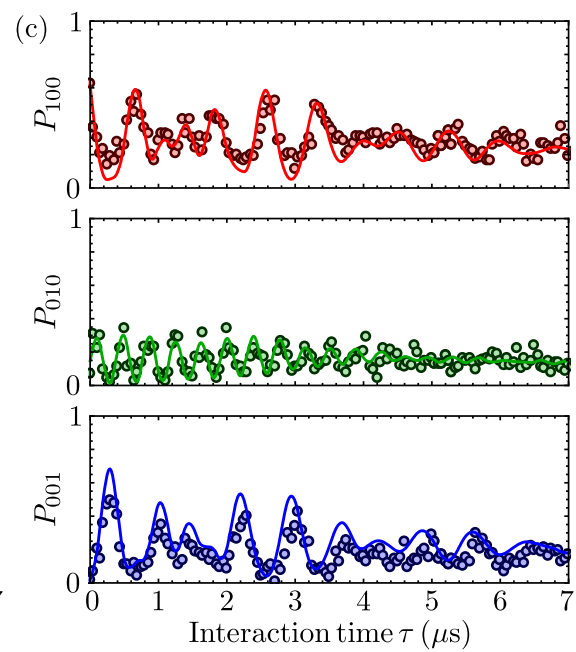

FIG. 3 (color online). Spin excitation transfer along a chain of three Rydberg atoms with nearest-neighbor separation of $20 \mu \mathrm{m}$. (a) Theoretical dynamics for a system initially prepared in $|\uparrow \downarrow \downarrow\rangle$, and evolving under a Hamiltonian similar to (1), but with only nearestneighbor interactions. (b) The same as (a), but for the full Hamiltonian (1), including long-range interactions. (c) Experimental data (points) and prediction of the model taking into account experimental imperfections (see text), with no adjustable parameters. For perfect preparation and readout, the probabilities $P_{\uparrow \downarrow \downarrow}$ (respectively, $P_{\downarrow \uparrow \downarrow}, P_{\downarrow \downarrow \uparrow}$ ) and $P_{100}$ (respectively, $P_{010}, P_{001}$ ) would coincide. 
Imperfect preparation and readout stem from the fact that, in addition to the spontaneous emission via the intermediate state during the optical pulses, the Rabi frequency for optical excitation $(\simeq 5.3 \mathrm{MHz})$ of atom 1 from $|g\rangle$ to $|\uparrow\rangle$ is not much higher than the interaction $(\simeq 0.92 \mathrm{MHz}$ for $R=20 \mu \mathrm{m})$. Thus, during the excitation of atom 1, the spin excitation already has a significant probability to hop to atom 2 . The damping essentially arises from the finite temperature of the atoms, which leads to changes in the interatomic distances, and thus in the couplings.

To go beyond this qualitative understanding of the limitations of our "quantum simulator," we add all known experimental imperfections to the $X Y$ model [28]. The result, shown by solid lines on Fig. 3(c) accurately reproduces the data with no free parameters. To obtain these curves, we simulate the full sequence, i.e., all three optical (de-) excitation pulses with or without the addressing beam, the microwave pulse, and evolution time, by solving the optical Bloch equations describing the dynamics of the internal states of the atoms, restricted to three states: $|g\rangle,|\uparrow\rangle$, and $|\downarrow\rangle$. Dissipation comes from both offresonant excitation of the intermediate $\left|5 P_{1 / 2}\right\rangle$ state during the optical excitation pulse, and from the finite lifetimes of the Rydberg states (101 and $135 \mu \mathrm{s}$ for $|\uparrow\rangle$ and $|\downarrow\rangle$, respectively [35]). The former effect is treated as an effective damping of the $|g\rangle \leftrightarrow|\uparrow\rangle$ transition, present only during the optical pulses, and with a damping rate chosen to match the damping of single-atom Rabi oscillations performed to calibrate the excitation Rabi frequency $\Omega_{\mathrm{opt}}[17]$.

We then account for the thermal motion of the atoms. A first consequence of the finite temperature $(T \simeq 50 \mu \mathrm{K})$ is that at the beginning of the sequence, the atoms have random positions (the transverse rms extension of the thermal motion in each microtrap, of radial frequency $90 \mathrm{kHz}$, is about $120 \mathrm{~nm}$ ) and random velocities (70 nm/ $\mu \mathrm{s} \mathrm{rms).} \mathrm{During} \mathrm{the} \mathrm{sequence,} \mathrm{the} \mathrm{traps} \mathrm{are} \mathrm{switched}$ off and the atoms are thus in free flight with their initial velocity. When solving the optical Bloch equations, we thus first draw the initial positions $\boldsymbol{r}_{i}^{0}$ and velocities $\boldsymbol{v}_{i}^{0}$ of each atom $i$ according to a thermal distribution, and use timedependent dipolar couplings $C_{3} /\left|\left(\boldsymbol{r}_{i}^{0}+\boldsymbol{v}_{i}^{0} t\right)-\left(\boldsymbol{r}_{j}^{0}+\boldsymbol{v}_{j}^{0} t\right)\right|^{3}$ in Eq. (1) [36]. We then average the results over 100 realizations. This yields a dephasing of the oscillations, resulting in a significant contrast reduction at long times.

A second effect of the temperature is that an atom has a small probability $\varepsilon(t)$ to leave the trap region during the experiment. In this case, we mistakenly infer that it was in a Rydberg state at the end of the sequence. This leads to a small distortion of the measured populations $P_{i j k}$ $(i, j, k=0,1)$ [37], that we compute from the actual ones as described in [17]. We measure $\varepsilon(t)$ (which increases with the duration $t$ of the sequence, from $\sim 1 \%$ at $t=0$ up to $\sim 20 \%$ for $t=7 \mu \mathrm{s}$ ) in a calibration experiment, and then

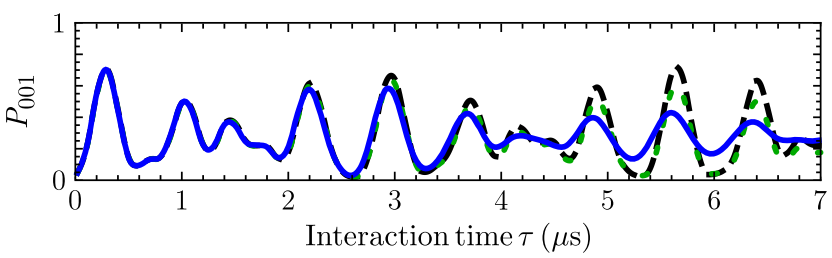

FIG. 4 (color online). Influence of the temperature on $P_{001}(\tau)$ : simulated dynamics at zero temperature (black dashed line), and adding either only atom loss (green dotted line), or only atomic motion (blue solid line).

use it to calculate the expected populations from the simulated ones [28].

Figure 4 shows how those two consequences of the finite temperature contribute to the observed damping in the dynamics of $P_{001}$ : both have sizable effects, but the dephasing due to fluctuations in the coupling dominates at long times. Reducing the atomic temperature using, e.g., Raman cooling [38,39] would render those effects negligible for our time scales, and allow the realization of a nearly ideal quantum simulator of spin dynamics.

In summary, we have measured the dynamics of a spin excitation in a minimal spin chain of three Rydberg atoms. The evolution of the system is accurately described by an $X Y$ Hamiltonian without any adjustable parameters. The obtained results are encouraging in view of scaling up the system to a larger number of spins. In particular, the residual motion of the atoms and the level of detection errors would already allow us to observe unambiguously the back-and-forth propagation of an excitation over a chain of $\sim 20$ atoms [28]. However, so far, experiments with more than $\sim 5$ atoms are hampered by the stochastic loading of the traps by single atoms [31]. In future work, we will thus explore various quasideterministic loading schemes that have been demonstrated at the level of a single $[40,41]$ or a few $[42,43]$ traps. Once this is achieved, our system will allow us to study the equivalent of an assembly of hard-core bosons on a 2D lattice with longrange, anisotropic hopping. We will also study dipolar interactions involving more than only two Rydberg states at an electrically tuned Förster resonance [44]. Our system will be ideal to study exotic phases and frustration in quantum magnetism, excitation hopping in complex networks $[45,46]$, or quantum walks with long-range hopping [47].

We acknowledge financial support by the EU [ERC Stg. Grant ARENA, AQUTE Integrating Project, FET-Open Xtrack Project HAIRS, and EU Marie-Curie Program ITN COHERENCE FP7-PEOPLE-2010-ITN-265031 (H. L.)], and by Région Île-de-France (LUMAT and Triangle de la Physique, projects LAGON and COLISCINA during the stay of C.S. A. at LCF). C.S. A. also acknowledges support from the U.K. EPSRC and Durham University. 
[1] A. Auerbach, Interacting Electrons and Quantum Magnetism (Springer-Verlag, New York, 1994).

[2] E. Collini, Chem. Soc. Rev. 42, 4932 (2013).

[3] M. Lewenstein, A. Sanpera, and V. Ahufinger, Ultracold Atoms in Optical Lattices: Simulating Quantum Many-Body Systems (Oxford University Press, Oxford, U.K, 2012).

[4] J. Simon, W. S. Bakr, R. Ma, M. E. Tai, P. M. Preiss, and M. Greiner, Nature (London) 472, 307 (2011).

[5] T. Fukuhara, P. Schauß, M. Endres, S. Hild, M. Cheneau, I. Bloch, and C. Gross, Nature (London) 502, 76 (2013).

[6] A. de Paz, A. Sharma, A. Chotia, E. Maréchal, J. H. Huckans, P. Pedri, L. Santos, O. Gorceix, L. Vernac, and B. Laburthe-Tolra, Phys. Rev. Lett. 111, 185305 (2013).

[7] B. Yan, S. A. Moses, B. Gadway, J. P. Covey, K. R. A. Hazzard, A. M. Rey, D. S. Jin, and J. Ye, Nature (London) 501, 521 (2013).

[8] K. Kim, M. S. Chang, S. Korenblit, R. Islam, E. E. Edwards, J. K. Freericks, G. D. Lin, L. M. Duan, and C. Monroe, Nature (London) 465, 590 (2010).

[9] P. Richerme, Z.-X. Gong, A. Lee, C. Senko, J. Smith, M. Foss-Feig, S. Michalakis, A. V. Gorshkov, and C. Monroe, Nature (London) 511, 198 (2014).

[10] P. Jurcevic, B. P. Lanyon, P. Hauke, C. Hempel, P. Zoller, R. Blatt, and C. F. Roos, Nature (London) 511, 202 (2014).

[11] P. Hauke, F. M. Cucchietti, A. Müller-Hermes, M.-C. Bañuls, J. I. Cirac, and M. Lewenstein, New J. Phys. 12, 113037 (2010).

[12] D. Peter, S. Müller, S. Wessel, and H. P. Büchler, Phys. Rev. Lett. 109, 025303 (2012).

[13] K. R. A. Hazzard, M. van den Worm, M. Foss-Feig, S. R. Manmana, E. Dalla Torre, T. Pfau, M. Kastner, and A. M. Rey, Phys. Rev. A 90, 063622 (2014).

[14] M. Avellino, A. J. Fisher, and S. Bose, Phys. Rev. A 74, 012321 (2006).

[15] M. Saffman, T. G. Walker, and K. Mølmer, Rev. Mod. Phys. 82, 2313 (2010).

[16] H. Weimer, M. Müller, I. Lesanovsky, P. Zoller, and H.-P. Büchler, Nat. Phys. 6, 382 (2010).

[17] D. Barredo, S. Ravets, H. Labuhn, L. Béguin, A. Vernier, F. Nogrette, T. Lahaye, and A. Browaeys, Phys. Rev. Lett. 112, 183002 (2014).

[18] P. Schauß, J. Zeiher, T. Fukuhara, S. Hild, M. Cheneau, T. Macrì, T. Pohl, I. Bloch, and C. Gross, arXiv:1404.0980.

[19] H. Weimer, R. Löw, T. Pfau, and H. P. Büchler, Phys. Rev. Lett. 101, 250601 (2008).

[20] T. Pohl, E. Demler, and M. D. Lukin, Phys. Rev. Lett. 104, 043002 (2010).

[21] I. Lesanovsky, Phys. Rev. Lett. 106, 025301 (2011).

[22] I. Mourachko, D. Comparat, F. de Tomasi, A. Fioretti, P. Nosbaum, V. M. Akulin, and P. Pillet, Phys. Rev. Lett. 80, 253 (1998).

[23] W. R. Anderson, J. R. Veale, and T. F. Gallagher, Phys. Rev. Lett. 80, 249 (1998).

[24] C. S. E. van Ditzhuijzen, A. F. Koenderink, J. V. Hernández, F. Robicheaux, L. D. Noordam, and H. B. van Linden van den Heuvell, Phys. Rev. Lett. 100, 243201 (2008).
[25] G. Günter, H. Schempp, M. Robert-de-Saint-Vincent, V. Gavryusev, S. Helmrich, C. S. Hofmann, S. Whitlock, and M. Weidemüller, Science 342, 954 (2013).

[26] S. Bettelli, D. Maxwell, T. Fernholz, C. S. Adams, I. Lesanovsky, and C. Ates, Phys. Rev. A 88, 043436 (2013).

[27] D. Maxwell, D. J. Szwer, D. Paredes-Barato, H. Busche, J. D. Pritchard, A. Gauguet, K. J. Weatherill, M. P. A. Jones, and C. S. Adams, Phys. Rev. Lett. 110, 103001 (2013).

[28] See Supplemental Material at http://link.aps.org/ supplemental/10.1103/PhysRevLett.114.113002, which includes Ref. [29], for extra details about the simulation of the spin chain dynamics.

[29] C. Tuchendler, A. M. Lance, A. Browaeys, Y. R. P. Sortais, and P. Grangier, Phys. Rev. A 78, 033425 (2008).

[30] L. Béguin, A. Vernier, R. Chicireanu, T. Lahaye, and A. Browaeys, Phys. Rev. Lett. 110, 263201 (2013).

[31] F. Nogrette, H. Labuhn, S. Ravets, D. Barredo, L. Béguin, A. Vernier, T. Lahaye, and A. Browaeys, Phys. Rev. X 4, 021034 (2014).

[32] The stray electric field is below $5 \mathrm{mV} / \mathrm{cm}$. Note that as (1) conserves the excitation number, the system is quite immune to decoherence due to global fluctuations in electric or magnetic fields.

[33] H. Labuhn, S. Ravets, D. Barredo, L. Béguin, F. Nogrette, T. Lahaye, and A. Browaeys, Phys. Rev. A 90, 023415 (2014).

[34] A. Reinhard, T. C. Liebisch, B. Knuffman, and G. Raithel, Phys. Rev. A 75, 032712 (2007).

[35] I. I. Beterov, I. I. Ryabtsev, D. B. Tretyakov, and V. M. Entin, Phys. Rev. A 79, 052504 (2009).

[36] Here, we neglect mechanical effects due to the interactions between Rydberg atoms during the interaction time. Using a classical model we estimate that these induce a displacement in $R$ below $30 \mathrm{~nm}$ for $\tau \leq 7 \mu$ s and are therefore negligible.

[37] C. Shen and L.-M. Duan, New J. Phys. 14, 053053 (2012).

[38] A. M. Kaufman, B. J. Lester, and C. A. Regal, Phys. Rev. X 2, 041014 (2012).

[39] J. D. Thompson, T. G. Tiecke, A. S. Zibrov, V. Vuletić, and M. D. Lukin, Phys. Rev. Lett. 110, 133001 (2013).

[40] T. Grünzweig, A. Hilliard, M. McGovern, and M. F. Andersen, Nat. Phys. 6, 951 (2010).

[41] M. Ebert, A. Gill, M. Gibbons, X. Zhang, M. Saffman, and T. G. Walker, Phys. Rev. Lett. 112, 043602 (2014).

[42] Y. Miroshnychenko, W. Alt, I. Dotsenko, L. Förster, M. Khudaverdyan, D. Meschede, D. Schrader, and A. Rauschenbeutel, Nature (London) 442, 151 (2006).

[43] M. Schlosser, J. Kruse, C. Gierl, S. Teichmann, S. Tichelmann, and G. Birkl, New J. Phys. 14, 123034 (2012).

[44] S. Ravets, H. Labuhn, D. Barredo, L. Béguin, T. Lahaye, and A. Browaeys, Nat. Phys. 10, 914 (2014).

[45] S. Wüster, C. Ates, A. Eisfeld, and J. M. Rost, Phys. Rev. Lett. 105, 053004 (2010).

[46] S. Mostarda, F. Levi, D. Prada-Gracia, F. Mintert, and F. Rao, arXiv:1312.1833.

[47] R. Côté, A. Russell, E. E. Eyler, and P. L. Gould, New J. Phys. 8, 156 (2006). 